\title{
Approximation of Analytic Functions by Solutions of Cauchy-Euler Equation
}

\author{
Soon-Mo Jung \\ Mathematics Section, College of Science and Technology, Hongik University, Sejong 30016, Republic of Korea \\ Correspondence should be addressed to Soon-Mo Jung; smjung@hongik.ac.kr
}

Received 2 November 2015; Accepted 15 May 2016

Academic Editor: Gestur Ólafsson

Copyright (c) 2016 Soon-Mo Jung. This is an open access article distributed under the Creative Commons Attribution License, which permits unrestricted use, distribution, and reproduction in any medium, provided the original work is properly cited.

We investigate the approximation properties of a special class of twice continuously differentiable functions by solutions of the Cauchy-Euler equation.

\section{Introduction}

Throughout this paper, let $n$ be a positive integer, let $I$ be a nondegenerate interval of $\mathbb{R}$, and let $\mathbb{K}$ denote either $\mathbb{R}$ or $\mathbb{C}$. We will consider the (linear) differential equation of $n$th order

$$
\mathscr{F}\left(y^{(n)}, y^{(n-1)}, \ldots, y^{\prime}, y, x\right)=0
$$

defined on $I$, where $y: I \rightarrow \mathbb{K}$ is an $n$ times continuously differentiable function.

For arbitrary $\varepsilon>0$, assume that an $n$ times continuously differentiable function $y: I \rightarrow \mathbb{K}$ satisfies the differential inequality

$$
\left|\mathscr{F}\left(y^{(n)}, y^{(n-1)}, \ldots, y^{\prime}, y, x\right)\right| \leq \varepsilon
$$

for all $x \in I$. If for each function $y: I \rightarrow \mathbb{K}$ satisfying inequality (2) there exists a solution $y_{0}: I \rightarrow \mathbb{K}$ of the differential equation (1) such that

$$
\left|y(x)-y_{0}(x)\right| \leq K(\varepsilon)
$$

for any $x \in I$, where $K(\varepsilon)$ depends on $\varepsilon$ only and satisfies $\lim _{\varepsilon \rightarrow 0} K(\varepsilon)=0$, then we say that the differential equation (1) satisfies (or has) the Hyers-Ulam stability (or the local HyersUlam stability if the domain $I$ is not the whole space $\mathbb{R})$. If the above statement also holds when we replace $\varepsilon$ and $K(\varepsilon)$ with some appropriate $\varphi(x)$ and $\Phi(x)$, respectively, then we say that the differential equation (1) satisfies the generalized Hyers-Ulam stability (or the Hyers-Ulam-Rassias stability).
We may apply these terminologies for other differential equations. For more detailed definition of the Hyers-Ulam stability and recent papers on this subject, refer to [1-4].

Obłoza seems to be the first author who investigated the Hyers-Ulam stability of linear differential equations (see $[5,6])$. Let $g, r:(a, b) \rightarrow \mathbb{R}$ be continuous functions with $\int_{a}^{b}|g(x)| d x<\infty$, where $a$ and $b$ are real constants. Assume that $\varepsilon>0$ is an arbitrary real number. Obłoza proved that if a differentiable function $y:(a, b) \rightarrow \mathbb{R}$ satisfies the inequality $\left|y^{\prime}(x)+g(x) y(x)-r(x)\right| \leq \varepsilon$ for all $x \in(a, b)$ and if a function $y_{0}:(a, b) \rightarrow \mathbb{R}$ satisfies $y_{0}^{\prime}(x)+g(x) y_{0}(x)=r(x)$ for all $x \in(a, b)$ and $y(\tau)=y_{0}(\tau)$ for some $\tau \in(a, b)$, then there exists a constant $\delta>0$ such that $\left|y(x)-y_{0}(x)\right| \leq \delta$ for all $x \in(a, b)$.

Thereafter, Alsina and Ger [7] proved that if a differentiable function $f:(a, b) \rightarrow \mathbb{R}$ satisfies the differential inequality $\left|y^{\prime}(x)-y(x)\right| \leq \varepsilon$, then there exists a solution $f_{0}$ : $(a, b) \rightarrow \mathbb{R}$ of the differential equation $y^{\prime}(x)=y(x)$ such that $\left|f(x)-f_{0}(x)\right| \leq 3 \varepsilon$ for any $x \in(a, b)$. This result of Alsina and Ger was generalized by Takahasi et al. They proved in [8] that the Hyers-Ulam stability holds for the Banach space valued differential equation $y^{\prime}(x)=\lambda y(x)$ (see also [9-13]). For a recent result on the Hyers-Ulam stability for second-order linear differential equations, we refer to $[14,15]$.

In this paper, we consider the (inhomogeneous) CauchyEuler equation

$$
x^{2} y^{\prime \prime}(x)+\alpha x y^{\prime}(x)+\beta y(x)=r(x)
$$


where $\alpha$ and $\beta$ are real-valued constants and $r: \mathbb{R} \rightarrow \mathbb{R}$ is a continuous function, and we investigate the approximation properties of twice continuously differentiable functions by solutions of the Cauchy-Euler equation

$$
x^{2} y^{\prime \prime}(x)+\alpha x y^{\prime}(x)+\beta y(x)=0,
$$

which is associated with (4).

\section{Preliminaries}

Recently, Choi and Jung [16, Corollary 4.2] proved the HyersUlam stability of the Cauchy-Euler equation (4) for the case of $(\alpha-1)^{2}-4 \beta>0$.

Theorem 1. Assume that the real-valued constants $\alpha, \beta$ are given with $(\alpha-1)^{2}-4 \beta>0$ and $\varepsilon$ is an arbitrarily given positive constant. Letc be a positive real-valued constant and let $m_{1}, m_{2}$ be given as

$$
\begin{aligned}
& m_{1}=\frac{-(\alpha-1)-\sqrt{(\alpha-1)^{2}-4 \beta}}{2}, \\
& m_{2}=\frac{-(\alpha-1)+\sqrt{(\alpha-1)^{2}-4 \beta}}{2} .
\end{aligned}
$$

If $r:(0, \infty) \rightarrow \mathbb{R}$ is a differentiable function and $y:(0, \infty) \rightarrow$ $\mathbb{R}$ is a twice continuously differentiable function such that the inequality

$$
\left|x^{2} y^{\prime \prime}(x)+\alpha x y^{\prime}(x)+\beta y(x)-r(x)\right| \leq \varepsilon
$$

holds for any $x \in(0, \infty)$, then there exists a solution $y_{c}$ : $(0, \infty) \rightarrow \mathbb{R}$ of the inhomogeneous Cauchy-Euler equation (4) such that

$$
\left|y(x)-y_{c}(x)\right| \leq \begin{cases}\frac{\varepsilon}{m_{1} m_{2}}+\frac{\varepsilon}{m_{2}-m_{1}}\left(\frac{1}{m_{2}}\left(\frac{x}{c}\right)^{m_{2}}-\frac{1}{m_{1}}\left(\frac{x}{c}\right)^{m_{1}}\right) & \left(\text { for } m_{1} \neq 0 \neq m_{2}\right), \\ \frac{\varepsilon}{m_{2}^{2}}\left(\left(\frac{x}{c}\right)^{m_{2}}-1\right)-\frac{\varepsilon}{m_{2}} \ln \frac{x}{c} & \left(\text { for } m_{1}=0\right), \\ \frac{\varepsilon}{m_{1}^{2}}\left(\left(\frac{x}{c}\right)^{m_{1}}-1\right)-\frac{\varepsilon}{m_{1}} \ln \frac{x}{c} & \left(\text { for } m_{2}=0\right)\end{cases}
$$

for all $x \in(0, \infty)$.

For the case of $(\alpha-1)^{2}-4 \beta=0$, the Hyers-Ulam stability of the inhomogeneous Cauchy-Euler equation (4) was proved in [16, Corollary 4.4].

Theorem 2. Assume that the real-valued constants $\alpha$ and $\beta$ are given with $\alpha \neq 1, \beta=(\alpha-1)^{2} / 4$ and $\varepsilon$ is an arbitrarily given positive constant. Let $c$ be a positive real-valued constant and let $\lambda=-(\alpha-1) / 2$. If $r:(0, \infty) \rightarrow \mathbb{R}$ is a differentiable function and $y:(0, \infty) \rightarrow \mathbb{R}$ is a twice continuously differentiable function such that the inequality

$$
\left|x^{2} y^{\prime \prime}(x)+\alpha x y^{\prime}(x)+\frac{(\alpha-1)^{2}}{4} y(x)-r(x)\right| \leq \varepsilon
$$

holds for all $x \in(0, \infty)$, then there exists a solution $y_{c}$ : $(0, \infty) \rightarrow \mathbb{R}$ of the inhomogeneous Cauchy-Euler equation (4) with $\beta=(\alpha-1)^{2} / 4$ such that

$$
\left|y(x)-y_{c}(x)\right| \leq \frac{\varepsilon}{\lambda^{2}}+\frac{\varepsilon}{\lambda}\left(\frac{x}{c}\right)^{\lambda}\left(\ln \frac{x}{c}-\frac{1}{\lambda}\right)
$$

for all $x \in(0, \infty)$.

Finally, the Hyers-Ulam stability of the Cauchy-Euler equation (4) was also proven in [16, Theorem 4.5] for the case of $(\alpha-1)^{2}-4 \beta<0$.
Theorem 3. Assume that the real-valued constants $\alpha$ and $\beta$ are given with $(\alpha-1)^{2}-4 \beta<0$ and $\varepsilon$ is an arbitrarily given positive constant. Let $c>0$ be a given real-valued constant and let

$$
\begin{aligned}
& \lambda=-\frac{\alpha-1}{2}, \\
& \mu=\frac{1}{2} \sqrt{4 \beta-(\alpha-1)^{2}} .
\end{aligned}
$$

If a differentiable function $r:(0, \infty) \rightarrow \mathbb{R}$ and a twice continuously differentiable function $y:(0, \infty) \rightarrow \mathbb{R}$ satisfy inequality (7) for all $x \in(0, \infty)$, then there exists a solution $y_{c}:(0, \infty) \rightarrow$ $\mathbb{R}$ of the inhomogeneous Cauchy-Euler equation (4) such that

$$
\left|y(x)-y_{c}(x)\right| \leq \frac{\varepsilon}{\mu}\left|\int_{c}^{x} \frac{x^{\lambda}}{\zeta^{\lambda+1}}\right| \sin \left(\mu \ln \frac{x}{\zeta}\right)|d \zeta|
$$

for all $x \in(0, \infty)$.

Remark 4. Cîmpean and Popa [14] proved the Hyers-Ulam stability of the linear differential equations of $n$th order with constant coefficients. Indeed, they proved a general theorem for the Hyers-Ulam stability which includes Theorems 1, 2, and 3 as its corollaries with the inequality 


$$
\left|y(x)-y_{c}(x)\right| \leq \begin{cases}\frac{\varepsilon}{|\beta|} & \left(\text { for either } \alpha^{2}-4 \beta>0, \beta \neq 0 \text { or } \alpha^{2}-4 \beta=0, \alpha \neq 0\right), \\ \frac{4 \varepsilon}{\alpha^{2}} & \left(\text { for } \alpha^{2}-4 \beta<0, \alpha \neq 0\right) .\end{cases}
$$

However, Theorems 1, 2, and 3 have the advantage of more exact local approximation over the result of Cîmpean and Popa as we see in Theorems 5, 6, and 7.

\section{Approximation Properties}

We denote by $\mathscr{B}(\alpha ; \beta)$ the set of all twice continuously differentiable functions $y:(0, \infty) \rightarrow \mathbb{R}$ for which there exists a constant $\varepsilon>0$ such that

$$
\left|x^{2} y^{\prime \prime}(x)+\alpha x y^{\prime}(x)+\beta y(x)\right| \leq \varepsilon
$$

for all $x \in(0, \infty)$, where $\alpha$ and $\beta$ are real-valued constants.

If we define

$$
\begin{aligned}
\left(y_{1}+y_{2}\right)(x) & :=y_{1}(x)+y_{2}(x), \\
\left(\gamma y_{1}\right)(x) & :=\gamma y_{1}(x)
\end{aligned}
$$

for all $y_{1}, y_{2} \in \mathscr{B}(\alpha ; \beta)$ and $\gamma \in \mathbb{R}$, then $\mathscr{B}(\alpha ; \beta)$ is a vector space over $\mathbb{R}$. This fact implies that the set $\mathscr{B}(\alpha ; \beta)$ is large enough to be a vector space.
In the following theorems, we investigate approximation properties of functions of $\mathscr{B}(\alpha ; \beta)$ by solutions of the CauchyEuler equation (5).

Theorem 5. Let $c>0$ be a given real number and let $\alpha, \beta \in \mathbb{R}$ be given with $(\alpha-1)^{2}-4 \beta>0$. If $y \in \mathscr{B}(\alpha ; \beta)$, then there exists a solution $y_{c}:(0, \infty) \rightarrow \mathbb{R}$ of the Cauchy-Euler equation (5) such that

$$
\left|y(x)-y_{c}(x)\right|=o(|x-c|)
$$

as $x \rightarrow c$

Proof. We define $m_{1}$ and $m_{2}$ by the formulas given in Theorem 1; that is, $m_{1}$ and $m_{2}$ are the distinct roots of the indicial equation $m^{2}+(\alpha-1) m+\beta=0$. Since $(\alpha-1)^{2}-4 \beta>0$, we have $m_{1}<m_{2}$. Since $y \in \mathscr{B}(\alpha ; \beta)$, there exists a constant $\varepsilon>0$ such that inequality (14) holds for all $x \in(0, \infty)$.

According to Theorem 1 with $r(x) \equiv 0$, there exists a solution $y_{c}:(0, \infty) \rightarrow \mathbb{R}$ of the Cauchy-Euler equation (5) such that

$$
\left|y(x)-y_{c}(x)\right| \leq \begin{cases}\frac{\varepsilon}{m_{1} m_{2}}+\frac{\varepsilon}{m_{2}-m_{1}}\left(\frac{1}{m_{2}}\left(\frac{x}{c}\right)^{m_{2}}-\frac{1}{m_{1}}\left(\frac{x}{c}\right)^{m_{1}}\right) & \left(\text { for } m_{1} \neq 0 \neq m_{2}\right), \\ \frac{\varepsilon}{m_{2}^{2}}\left(\left(\frac{x}{c}\right)^{m_{2}}-1\right)-\frac{\varepsilon}{m_{2}} \ln \frac{x}{c} & \left(\text { for } m_{1}=0\right), \\ \frac{\varepsilon}{m_{1}^{2}}\left(\left(\frac{x}{c}\right)^{m_{1}}-1\right)-\frac{\varepsilon}{m_{1}} \ln \frac{x}{c} & \left(\text { for } m_{2}=0\right)\end{cases}
$$

for any $x \in(0, \infty)$.

We will only estimate the following limit for the case of $m_{1}=0$ by applying L'Hospital's rule:

$$
\begin{aligned}
\lim _{x \rightarrow c}\left|\frac{y(x)-y_{c}(x)}{x-c}\right| \\
\quad \leq\left|\lim _{x \rightarrow c} \frac{\left(\varepsilon / m_{2}^{2}\right)\left((x / c)^{m_{2}}-1\right)-\left(\varepsilon / m_{2}\right) \ln (x / c)}{x-c}\right| \\
\quad=\left|\lim _{x \rightarrow c}\left(\frac{\varepsilon}{m_{2} c}\left(\frac{x}{c}\right)^{m_{2}-1}-\frac{\varepsilon}{m_{2}} \frac{1}{x}\right)\right|=0,
\end{aligned}
$$

which implies the validity of this theorem.

We now consider the case of $(\alpha-1)^{2}-4 \beta=0$ and use Theorem 2 to prove the following theorem.
Theorem 6. Let $c>0$ and $\alpha \neq 1$ be real numbers and let $\lambda=-(\alpha-1) / 2$. If $y \in \mathscr{B}\left(\alpha ; \lambda^{2}\right)$, then there exists a solution $y_{c}:(0, \infty) \rightarrow \mathbb{R}$ of the Cauchy-Euler equation (5) with $\beta=\lambda^{2}$ such that

$$
\left|y(x)-y_{c}(x)\right|=o(|x-c|)
$$

as $x \rightarrow c$.

Proof. Since $y \in \mathscr{B}\left(\alpha ; \lambda^{2}\right)$, there exists a constant $\varepsilon>0$ such that inequality (14) holds for all $x \in(0, \infty)$. According to Theorem 2 with $r(x) \equiv 0$, there exists a solution $y_{c}:(0, \infty) \rightarrow$ $\mathbb{R}$ of the Cauchy-Euler equation (5) with $\beta=\lambda^{2}$ such that

$$
\left|y(x)-y_{c}(x)\right| \leq \frac{\varepsilon}{\lambda^{2}}+\frac{\varepsilon}{\lambda}\left(\frac{x}{c}\right)^{\lambda}\left(\ln \frac{x}{c}-\frac{1}{\lambda}\right)
$$

for all $x \in(0, \infty)$. 
Therefore, we estimate the limit by applying L'Hospital's rule:

$$
\begin{aligned}
\lim _{x \rightarrow c}\left|\frac{y(x)-y_{c}(x)}{x-c}\right| \\
\quad \leq\left|\lim _{x \rightarrow c} \frac{\left(\varepsilon / \lambda^{2}\right)+(\varepsilon / \lambda)(x / c)^{\lambda}(\ln (x / c)-1 / \lambda)}{x-c}\right| \\
\quad=\left|\lim _{x \rightarrow c} \varepsilon \frac{x^{\lambda-1}}{c^{\lambda}} \ln \frac{x}{c}\right|=0,
\end{aligned}
$$

which implies the validity of this theorem.

Finally, we investigate the approximation property of each function of $\mathscr{B}(\alpha ; \beta)$ by a solution of the differential equation (5) when $(\alpha-1)^{2}-4 \beta<0$.

Theorem 7. Let $c>0$ be a given real number and let $\alpha, \beta \in \mathbb{R}$ be given with $(\alpha-1)^{2}-4 \beta<0$. If $y \in \mathscr{B}(\alpha ; \beta)$, then there exists a solution $y_{c}:(0, \infty) \rightarrow \mathbb{R}$ of the Cauchy-Euler equation (5) such that

$$
\left|y(x)-y_{c}(x)\right|=o(|x-c|)
$$

as $x \rightarrow c$.

Proof. Let us define

$$
\begin{aligned}
& \lambda=-\frac{\alpha-1}{2}, \\
& \mu=\frac{1}{2} \sqrt{4 \beta-(\alpha-1)^{2}} .
\end{aligned}
$$

Since $y \in \mathscr{B}(\alpha ; \beta)$, there exists a constant $\varepsilon>0$ such that inequality (14) holds for all $x \in(0, \infty)$. According to Theorem 3 with $r(x) \equiv 0$, there exists a solution $y_{c}:(0, \infty) \rightarrow$ $\mathbb{R}$ of the Cauchy-Euler equation (5) such that

$$
\left|y(x)-y_{c}(x)\right| \leq \frac{\varepsilon}{\mu}\left|\int_{c}^{x} \frac{x^{\lambda}}{\zeta^{\lambda+1}}\right| \sin \left(\mu \ln \frac{x}{\zeta}\right)|d \zeta|
$$

for all $x \in(0, \infty)$.

If we substitute $\eta=c x / \zeta$, then we have

$$
\begin{aligned}
& \int_{c}^{x} \frac{x^{\lambda}}{\zeta^{\lambda+1}}\left|\sin \left(\mu \ln \frac{x}{\zeta}\right)\right| d \zeta \\
& =\frac{1}{c^{\lambda}} \int_{c}^{x} \eta^{\lambda-1}\left|\sin \left(\mu \ln \frac{\eta}{c}\right)\right| d \eta .
\end{aligned}
$$

Hence, we further apply L'Hospital's rule to obtain

$$
\begin{aligned}
\lim _{x \rightarrow c}\left|\frac{y(x)-y_{c}(x)}{x-c}\right| \\
\leq \frac{\varepsilon}{\mu}\left|\lim _{x \rightarrow c} \frac{1}{x-c} \int_{c}^{x} \frac{x^{\lambda}}{\zeta^{\lambda+1}}\right| \sin \left(\mu \ln \frac{x}{\zeta}\right)|d \zeta| \\
=\frac{\varepsilon}{\mu c^{\lambda}}\left|\lim _{x \rightarrow c} \frac{1}{x-c} \int_{c}^{x} \eta^{\lambda-1}\right| \sin \left(\mu \ln \frac{\eta}{c}\right)|d \eta| \\
=\frac{\varepsilon}{\mu c^{\lambda}}\left|\lim _{x \rightarrow c} x^{\lambda-1}\right| \sin \left(\mu \ln \frac{x}{c}\right)||=0,
\end{aligned}
$$

which implies the validity of this theorem.

\section{Competing Interests}

The author declares that there are no competing interests.

\section{Acknowledgments}

This research was supported by Basic Science Research Program through the National Research Foundation of Korea (NRF) funded by the Ministry of Education (no. 2013R1A1A2005557). This work was supported by 2014 Hongik University Research Fund.

\section{References}

[1] J.-H. Bae and W.-G. Park, "On the Ulam stability of the CauchyJensen equation and the additive-quadratic equation," Journal of Nonlinear Science and Its Applications, vol. 8, no. 5, pp. 710-718, 2015.

[2] D. H. Hyers, G. Isac, and Th. M. Rassias, Stability of Functional Equations in Several Variables, Birkhäuser, Boston, Mass, USA, 1998.

[3] S.-M. Jung, Hyers-Ulam-Rassias Stability of Functional Equations in Nonlinear Analysis, vol. 48 of Springer Optimization and Its Applications, Springer, New York, NY, USA, 2011.

[4] S. O. Kim, A. Bodaghi, and C. Park, "Stability of functional inequalities associated with the Cauchy-Jensen additive functional equalities in non-Archimedean Banach spaces," Journal of Nonlinear Science and Its Applications, vol. 8, no. 5, pp. 776786, 2015.

[5] M. Ob loza, "Hyers stability of the linear differential equation," Rocznik Naukowo-Dydaktyczny. Prace Matematyczne, no. 13, pp. 259-270, 1993.

[6] M. Obłoza, "Connections between Hyers and Lyapunov stability of the ordinary differential equations," Rocznik Nauk.Dydakt. Prace Mat Journals, vol. 14, pp. 141-146, 1997.

[7] C. Alsina and R. Ger, "On some inequalities and stability results related to the exponential function," Journal of Inequalities and Applications, vol. 2, no. 4, pp. 373-380, 1998.

[8] S.-E. Takahasi, T. Miura, and S. Miyajima, "On the Hyers-Ulam stability of the Banach space-valued differential equation $y^{\prime}=$ $\lambda y$," Bulletin of the Korean Mathematical Society, vol. 39, pp. 309-315, 2002.

[9] S.-M. Jung, "Hyers-ulam stability of linear differential equations of first order," Applied Mathematics Letters, vol. 17, no. 10, pp. 1135-1140, 2004.

[10] S.-M. Jung, "Hyers-Ulam stability of linear differential equations of first order, II," Applied Mathematics Letters, vol. 19, no. 9, pp. 854-858, 2006.

[11] D. Popa and I. Raşa, "On the Hyers-Ulam stability of the linear differential equation," Journal of Mathematical Analysis and Applications, vol. 381, no. 2, pp. 530-537, 2011.

[12] D. Popa and I. Raşa, "Hyers-Ulam stability of the linear differential operator with non-constant coefficients," Applied Mathematics and Computation, vol. 219, no. 4, pp. 1562-1568, 2012.

[13] G. Wang, M. Zhou, and L. Sun, "Hyers-Ulam stability of linear differential equations of first order," Applied Mathematics Letters, vol. 21, no. 10, pp. 1024-1028, 2008.

[14] D. S. Cîmpean and D. Popa, "On the stability of the linear differential equation of higher order with constant coefficients," 
Applied Mathematics and Computation, vol. 217, no. 8, pp. 41414146, 2010.

[15] Y. Li and Y. Shen, "Hyers-Ulam stability of linear differential equations of second order," Applied Mathematics Letters, vol. 23, no. 3, pp. 306-309, 2010.

[16] G. Choi and S.-M. Jung, "Invariance of Hyers-Ulam stability of linear differential equations and its applications," Advances in Difference Equations, vol. 2015, article 277, 14 pages, 2015. 


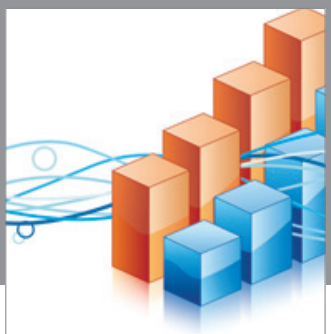

Advances in

Operations Research

vatem alat4

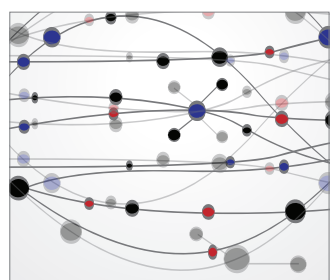

\section{The Scientific} World Journal
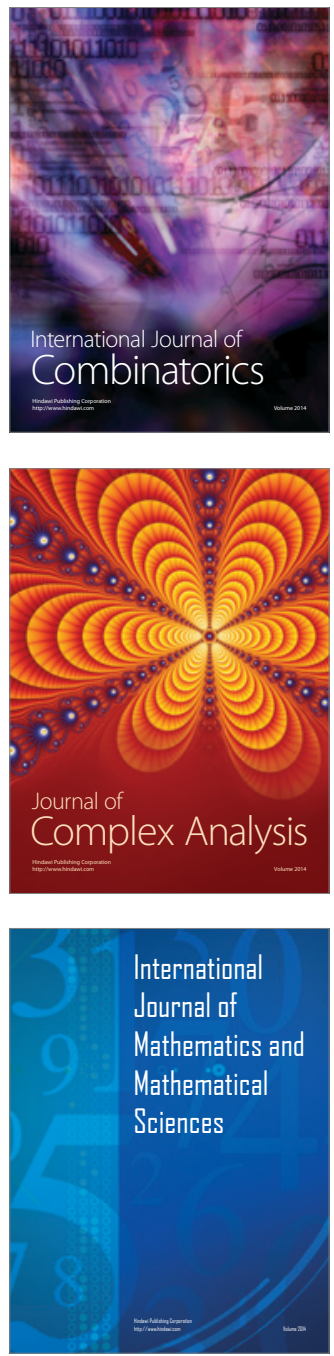
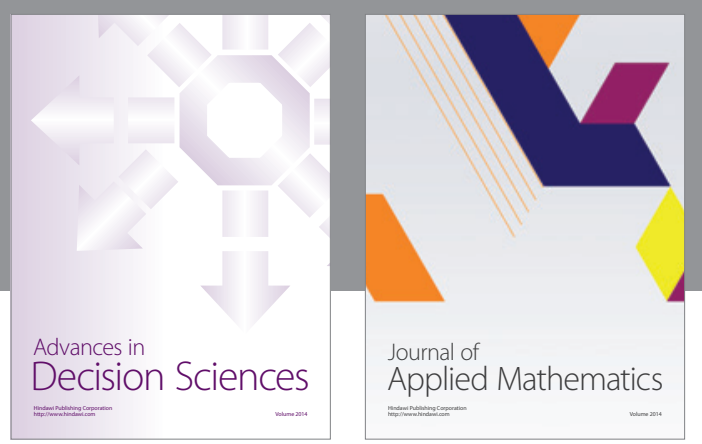

Algebra

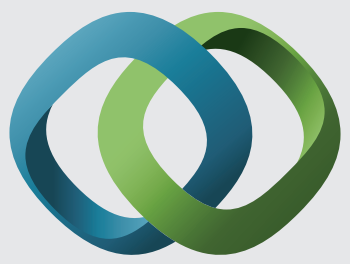

\section{Hindawi}

Submit your manuscripts at

http://www.hindawi.com
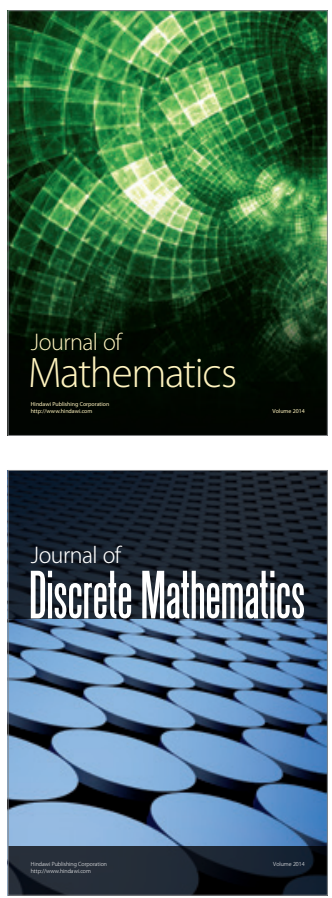

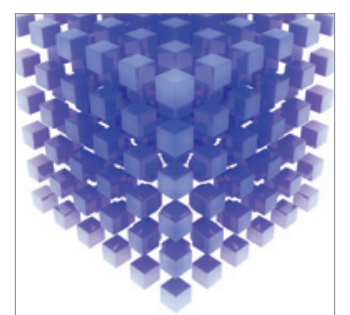

Mathematical Problems in Engineering
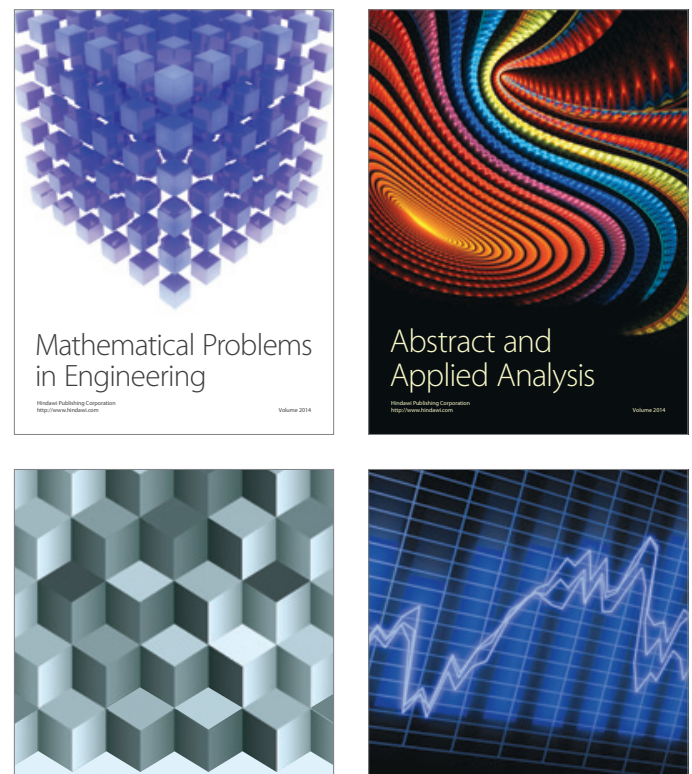

Journal of

Function Spaces

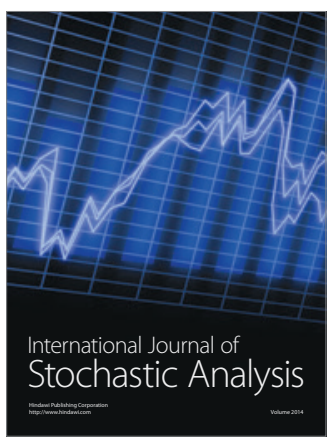

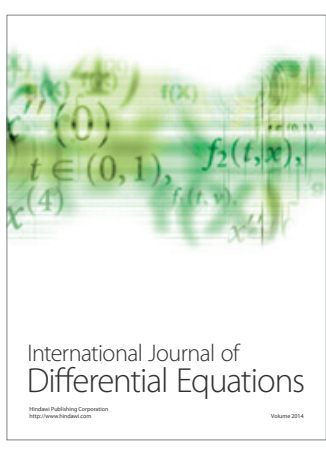
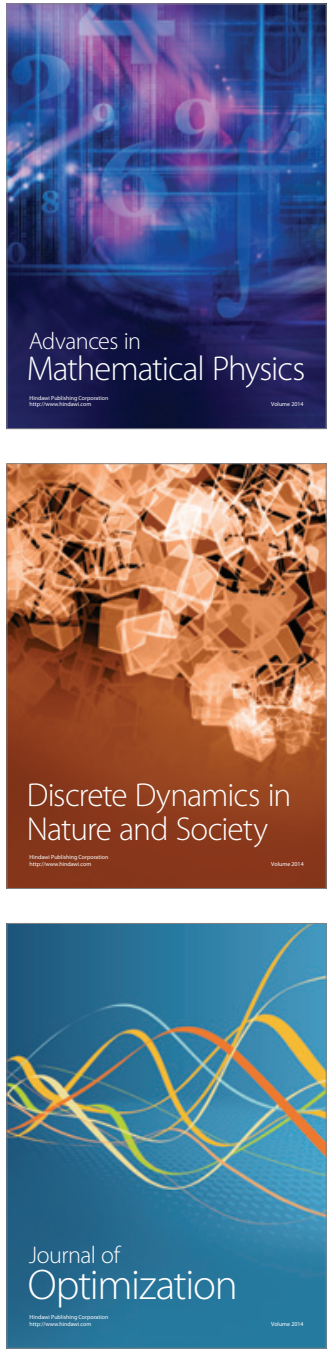